\title{
Characterization of Low-Strigolactone Germplasm in Pea (Pisum sativum L.) Resistant to Crenate Broomrape (Orobanche crenata Forsk.)
}

\author{
Stefano Pavan, ${ }^{1}$ Adalgisa Schiavulli, ${ }^{2}$ Angelo Raffaele Marcotrigiano, ${ }^{1}$ Nicoletta Bardaro, ${ }^{1}$ \\ Valentina Bracuto, ${ }^{1}$ Francesca Ricciardi, ${ }^{2}$ Tatsiana Charnikhova,${ }^{3}$ Concetta Lotti, ${ }^{2}$ \\ Harro Bouwmeester, ${ }^{3}$ and Luigi Ricciardi ${ }^{1}$ \\ ${ }^{1}$ Department of Plant, Soil and Food Science, Section of Genetics and Plant Breeding, University of Bari, via Amendola 165/A, \\ 70126 Bari, Italy; ${ }^{2}$ Department of Agriculture, Food and Environmental Science, University of Foggia, via Napoli 25, 71100 \\ Foggia, Italy; and ${ }^{3}$ Laboratory of Plant Physiology, Wageningen University, Droevendaalsesteeg 1, 6708 PB Wageningen, \\ The Netherlands
}

Accepted 17 June 2016.

\begin{abstract}
Crenate broomrape (Orobanche crenata Forsk.) is a devastating parasitic weed threatening the cultivation of legumes around the Mediterranean and in the Middle East. So far, only moderate levels of resistance were reported to occur in pea (Pisum sativum $\mathbf{L}$.) natural germplasm, and most commercial cultivars are prone to severe infestation. Here, we describe the selection of a pea line highly resistant to $O$. crenata, following the screening of local genetic resources. Time series observations show that delayed emergence of the parasite is an important parameter associated with broomrape resistance. High performance liquid chromatography connected to tandem mass spectrometry analysis and in vitro broomrape germination bioassays suggest that the resistance mechanism might involve the reduced secretion of strigolactones, plant hormones exuded by roots and acting as signaling molecules for the germination of parasitic weeds. Two years of replicated trials in noninfested fields indicate that the resistance is devoid of pleiotropic effects on yield, in contrast to pea experimental mutants impaired in strigolactone biosynthesis and, thus, is suitable for use in breeding programs.
\end{abstract}

Crenate broomrape (Orobanche crenata Forsk.) is a widespread parasitic weed occurring around the Mediterranean and in the Middle East. As most other species belonging to the botanic family of Orobanchaceae, $O$. crenata penetrates the roots of its hosts and establishes a vascular connection enabling it to drain water, nutrients, and assimilates. After developing underground tubercles, it emerges above soil with a flowering shoot and terminates its life cycle with the production of a large number of minute seeds that can remain viable for many years (Yoneyama et al. 2010).

Infestations of $O$. crenata are a serious constraint for many legumes and can be devastating for pea (Pisum sativum L.), the most widely grown grain legume in Europe and the fourth most in the world (Pavan et al. 2013). Indeed, serious infestations can result in a complete yield loss and relegate pea cultivation to

Corresponding author: S. Pavan; E-mail: stefano.pavan@uniba.it

*The $\boldsymbol{e}$-Xtra logo stands for "electronic extra" and indicates that one supplementary figure is published online.

(c) 2016 The American Phytopathological Society noninfested areas (Rubiales et al. 2009). Limited success in the control of $O$. crenata has been achieved by means of agronomic and chemical strategies. Therefore, the identification and practical exploitation of genotypes displaying resistance to $O$. crenata is one of the main aims of pea breeding (Rubiales et al. 2009).

Plant resistance is the result of mechanisms enabling the avoidance of parasite attack or, following attack, preventing the establishment and growth of the parasite (Joel et al. 2013). Concerning interactions between pea and $O$. crenata, defense strategies of the first type include reduced root biomass, deep rooting, short growing cycle, and low stimulation of parasite seed germination; defense strategies of the second type can occur during several developmental stages, such as host penetration, attachment to the xylem, and development of the tubercle (Perez De Luque et al. 2005). However, only low levels of resistance have been reported so far to occur in natural pea germplasm and most commercial pea cultivars are highly susceptible (Rubiales et al. 2003, 2005).

Strigolactones are a class of carotenoid-derived compounds secreted from plant roots into the rhizosphere. Decades ago, they were characterized as host recognition signals for root parasitic family Orobanchaceae, whose germination is stimulated upon strigolactone perception (Cook et al. 1966). More than eighteen strigolactones, differing in their germination stimulatory activity, have been identified in plants, and species differ considerably in the strigolactone pattern they secrete (Yoneyama et al. 2009). Three strigolactones have been identified in pea root exudates, i.e., orobanchol, orobanchyl acetate, and epoxyorobanchyl (fabacyl) acetate, with the latter having the highest biological activity toward the pea parasitic plant species Orobanche minor (Xie et al. 2009). More recently, additional roles were discovered for strigolactones as host recognition signals for symbiotic arbuscular mycorrhizal fungi and as phytohormones controlling different aspects of plant development, including the inhibition of shoot branching (Akiyama et al. 2005; Brewer et al. 2013; Gomez-Roldan et al. 2008; Ruyter-Spira et al. 2013; Umehara et al. 2008).

Considering the crucial function of strigolactones in the interaction between parasitic plants and their hosts, the selection of genotypes releasing low levels of strigolactones into the rhizosphere has been envisaged as a valuable breeding strategy to introduce resistance in crop species (Cardoso et al. 2011; Jamil et al. 2011). In pea, strigolactones were undetectable in lines with mutations in $R M S 5$ and $R M S 1$, two key genes in the 
strigolactone biosynthetic pathway that encode the enzymes carotenoid cleavage dioxygenase (CCD)7 and CCD8, respectively (Gomez-Roldan et al. 2008). Although such mutants were shown to induce markedly lower germination of $O$. crenata seeds, they display severe pleiotropic effects such as dwarfism and an extremely branched phenotype (Rameau et al. 1997; Gomez-Roldan et al. 2008). Therefore, they are not suitable to be used in breeding.

Here, we report the selection of a pea landrace displaying a high level of resistance to $O$. crenata. We show that the resistance is likely due to genetically determined lower production of strigolactones and could be used for breeding purposes.

\section{RESULTS}

\section{Identification and characterization of a source of resistance to $O$. crenata in pea germplasm.}

In May 2000, a garden pea landrace cultivated in the area of Bari, in the south of Italy, was noticed for showing no sign of O. crenata parasitism, in contrast to the genotypes of faba bean it was intercropped with. Several pure lines were originated from this landrace by repeated self-pollination and were further evaluated in an experimental field known to be infested with elevated levels of $O$. crenata seeds. Finally, a resistant line named ROR12 was selected (Fig. 1). In four different trials, carried out in infested fields during three different growing seasons (2009-2010, 2010-2011, and 2012-2013), ROR12 displayed much higher seed production compared with commercial garden pea cultivars used as controls, which suffered up to total yield loss (Table 1). In three of these four trials, the average number of emerged $O$. crenata shoots per host plant at crop maturity was significantly lower on ROR12 (ranging from 0 to 1.3 ) than in susceptible controls, while in one of the two trials carried out in 2012-2013 no significant difference was detected (Table 1). However, when time series observations for this trial were taken into account, it was realized that $O$. crenata emergence on ROR12 was significantly delayed compared with the susceptible controls and only occurred at the later phenological stages (Fig. 2).

\section{ROR12 has a good agronomic performance} under noninfested conditions.

In order to be useful in practical breeding, genetic resistance must be not coupled with pleiotropic phenotypes affecting the overall agronomic performance. Aiming to evaluate the

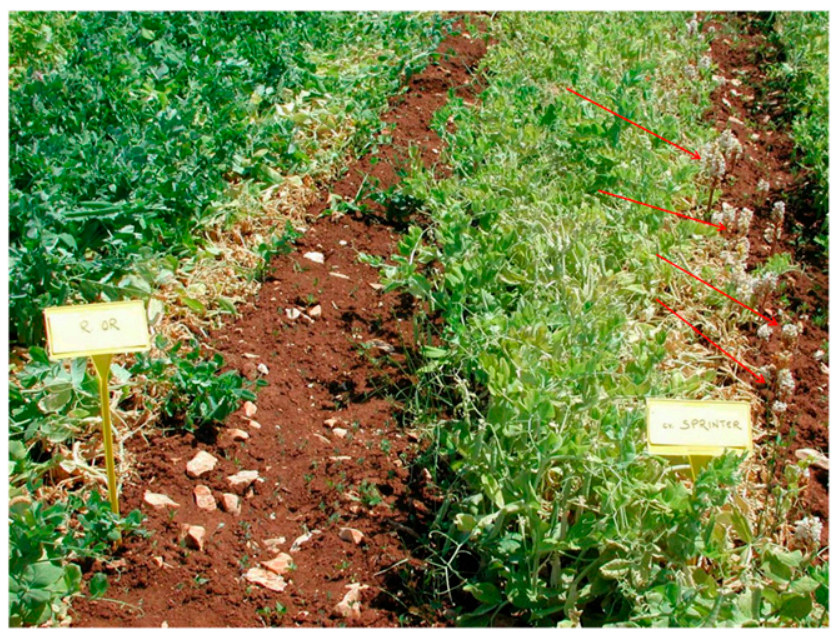

Fig. 1. Phenotypic response to Orobanche crenata of the ROR12 line selected in this study (left row) and 'Sprinter' (right row). Arrows indicate $O$. crenata emerged floral shoots. breeding value of the newly identified $O$. crenata resistance source, we carried out two additional field trials during the growing seasons 2011-2012 and 2012-2013, in which the ROR12 line and six commercial garden pea cultivars were grown at common planting density in noninfested experimental plots. In both trials, ROR12 yield (1.58 Mg/ha in 2011-2012 and $1.97 \mathrm{Mg} / \mathrm{ha}$ in 2012-2013) was not significantly different from the one of most other cultivars, indicating that it has a good agronomic potential independently from the presence of O. crenata (Fig. 3).

\section{ROR12 is a low-strigolactone line.}

Later emergence of $O$. crenata on ROR 12 suggests that its resistance might be due to delayed germination of parasitic weed seeds. This is induced by germination stimulants, the most prominent of which are the strigolactones. Moreover, we noticed that ROR 12 exhibits a more branched phenotype, and it has been demonstrated that strigolactones are involved together with auxins in the inhibition of shoot branching (GomezRoldan et al. 2008). Taken all this into account, we decided to test the hypothesis that ROR12 exudes lower levels of strigolactones. Therefore, high performance liquid chromatography connected to tandem mass spectrometry (LC-MS/MS) was performed to compare the strigolactone level in the ROR12 root exudate with that of the three susceptible cultivars Sprinter, NanoProgress9, and Dorian. In order to facilitate the detection of genotypic differences, exudates were collected from plants exposed to phosphorous starvation, which is known to enhance the biosynthesis of strigolactones (López-Ráez et al. 2008; Yoneyama et al. 2007). All the three strigolactones previously identified in pea root exudates (Xie et al. 2009) —orobanchol, orobanchyl acetate, and fabacyl acetate-were detected, after purification on a silica column, in fractions 20,40 , and $60 \%$ ethyl acetate in hexane (Supplementary Fig. S1). The level of all three was markedly lower in ROR 12 compared with the susceptible controls at all time points after the start of phosphorous starvation (5, 8, and 11 days) (Fig. 4). In the case of 'Sprinter', clear positive correlation was observed between duration of phosphorous starvation and levels of the three strigolactones. Although a similar trend could be noticed for the other susceptible genotypes under test ('Dorian' and 'NanoProgress9'), variation in strigolactone content was less obvious. Fabacyl acetate was the only strigolactone in ROR12 root exudates showing a moderate increase with time of phosphorous starvation.

Theoretically, the lower strigolactone content of ROR12 root exudates could be due either to reduced secretion rates per root weight unit or to reduced root biomass. To investigate this aspect, in a parallel experiment, we measured the root dry weight of 1-month-old ROR12, 'Sprinter', and 'NanoProgress9' plants grown in aereoponics culture. Dry root biomass of ROR12 (1.98 g) was not statistically different from that of 'Nanoprogress9' (1.92 g) and was significantly higher $(P<0.05)$ than that of 'Sprinter' $(1.74 \mathrm{~g})$. This supports the theory that reduced strigolactone levels in ROR 12 exudates are due to a reduced strigolactone biosynthesis or exudation and not to a lower root biomass.

\section{ROR12 root exudates have lower activity in stimulating $O$. crenata germination.}

The germination stimulant activity of ROR12 root exudate on $O$. crenata seeds was assessed and compared with the one of susceptible 'Sprinter', by means of a petri-dish bioassay. On all time points after the initiation of phosphorous starvation, 'Sprinter' root exudate induced higher $O$. crenata seed germination than ROR12 (Fig. 5). No germination was detected in water-treated seeds (data not shown). The same trend was 
observed when the root exudates were concentrated five-, 10- or 25-fold.

\section{DISCUSSION}

Here, we report the identification of a pea landrace highly resistant to $O$. crenata. This is a relevant finding, considering that former screenings of pea germplasm had limited success in detecting sources of broomrape resistance (Rubiales et al. 2009). Resistance was assessed under field conditions and ensured good yields even in situations of severe infestation. In one of the experimental trials, time series observations suggested that resistance was not associated with a reduction in the number of $O$. crenata shoots emerged aboveground at crop maturity but, rather, with a delayed emergence of the parasite. This evidence argues against the accurateness of previous investigations that exclusively scored the number of $O$. crenata shoots at crop maturity to evaluate genotypic breeding values (Rubiales et al. 2006). Moreover, it indicates the importance of delayed attachment or emergence of $O$. crenata as a mechanism enabling the plant to avoid severe yield loss (Fig. 2). In this respect, our findings substantiate those of Van Ast and Bastiaans (2006), who reported a dramatic increase of sorghum yield following a delay of two weeks in artificial infection with the root parasitic plant species Striga hermonthica.

Although ROR12 always showed higher yield than a panel of control cultivars in infested fields, in one of the trials, it displayed a similar number of $O$. crenata floral shoots at crop maturity (Fig. 2). This can likely be explained by the severity of infestation, which resulted in early wilting and death of many individuals of the (nonresistant) cultivars. Thus, during the last phase of the experiment, $O$. crenata emergence only occurred at a very low rate on the susceptible genotypes, while ROR12 sustained a higher infection or emergence rate.

Together, our LC-MS/MS analysis and in vitro broomrape germination bioassays (Figs. 4 and 5) make plausible the hypothesis that ROR12 resistance is due to the reduced secretion of strigolactones, plant hormones, and host recognition signals inducing the germination of parasitic plant seeds (Kohlen et al. 2012; Vogel et al. 2010). However, the experimental evidence

Table 1. Results of four field trials evaluating the phenotype of the Pisum sativum line ROR12 and several commercial cultivars in fields infested by Orobanche crenata

\begin{tabular}{|c|c|c|c|c|}
\hline \multirow[b]{2}{*}{ Genotype } & \multicolumn{2}{|c|}{ 2009-2010 } & \multicolumn{2}{|c|}{$2010-2011$} \\
\hline & O. crenata shoots & Yield/plant (g) & O. crenata shoots & Yield/plant (g) \\
\hline \multirow{4}{*}{$\begin{array}{l}\text { ROR12 } \\
\text { 'Sprinter' }\end{array}$} & $0 \pm 0$ & $75.3 \pm 6.5$ & $1.3 \pm 0.1$ & $41.8 \pm 1.8$ \\
\hline & $6.5 \pm 0.7 * *$ & $29.9 \pm 3.3 * *$ & $9.9 \pm 0.4 * *$ & $6.4 \pm 0.9 * *$ \\
\hline & \multicolumn{4}{|c|}{$2012-2013$} \\
\hline & \multicolumn{2}{|c|}{ 1st trial } & \multicolumn{2}{|c|}{ 2nd trial } \\
\hline Genotype & O. crenata shoots & Yield/plant (g) & O. crenata shoots & Yield/plant (g) \\
\hline ROR12 & $0.2 \pm 0.1$ & $31.4 \pm 4.2$ & $4.5 \pm 0.6$ & $35.2 \pm 2.5$ \\
\hline 'Sprinter' & $2.1 \pm 0.3 * *$ & $2.0 \pm 0.6^{* *}$ & $3.3 \pm 0.4^{\mathrm{n} . \mathrm{s}}$ & $0.9 \pm 0.2^{* *}$ \\
\hline 'Dorian' & $2.7 \pm 0.4 * *$ & $0.8 \pm 0.8^{* *}$ & $4.7 \pm 0.7^{\mathrm{n} . \mathrm{s}}$ & $2.2 \pm 0.5^{* *}$ \\
\hline 'NanoProgress9' & $2.6 \pm 0.3^{* *}$ & $1.7 \pm 0.6^{* *}$ & $2.7 \pm 0.5^{\mathrm{n} . \mathrm{s}}$ & $0.1 \pm 0.1 * *$ \\
\hline 'Onward' & $4.3 \pm 0.6^{* *}$ & $0.8 \pm 0.4^{* *}$ & $4.5 \pm 0.7^{\mathrm{n} . \mathrm{s}}$ & $0.2 \pm 0.1 * *$ \\
\hline 'Rondo' & $2.6 \pm 0.4 * *$ & $3.2 \pm 0.9^{* *}$ & $4.6 \pm 0.7^{\mathrm{n} . \mathrm{s}}$ & $0.3 \pm 0.1 * *$ \\
\hline 'Utrillo' & $2.5 \pm 0.4 * *$ & $0.6 \pm 0.4^{* *}$ & $4.9 \pm 0.7^{\mathrm{n} . \mathrm{s}}$ & $1.5 \pm 0.4 * *$ \\
\hline
\end{tabular}

a The number of $O$. crenata shoots per plant was recorded at crop maturity. Asterisks indicate significant differences, inferred by performing Student's $t$ tests $(P<0.01)$; n.s. indicates nonsignificant differences with ROR12 as the result of the same test.

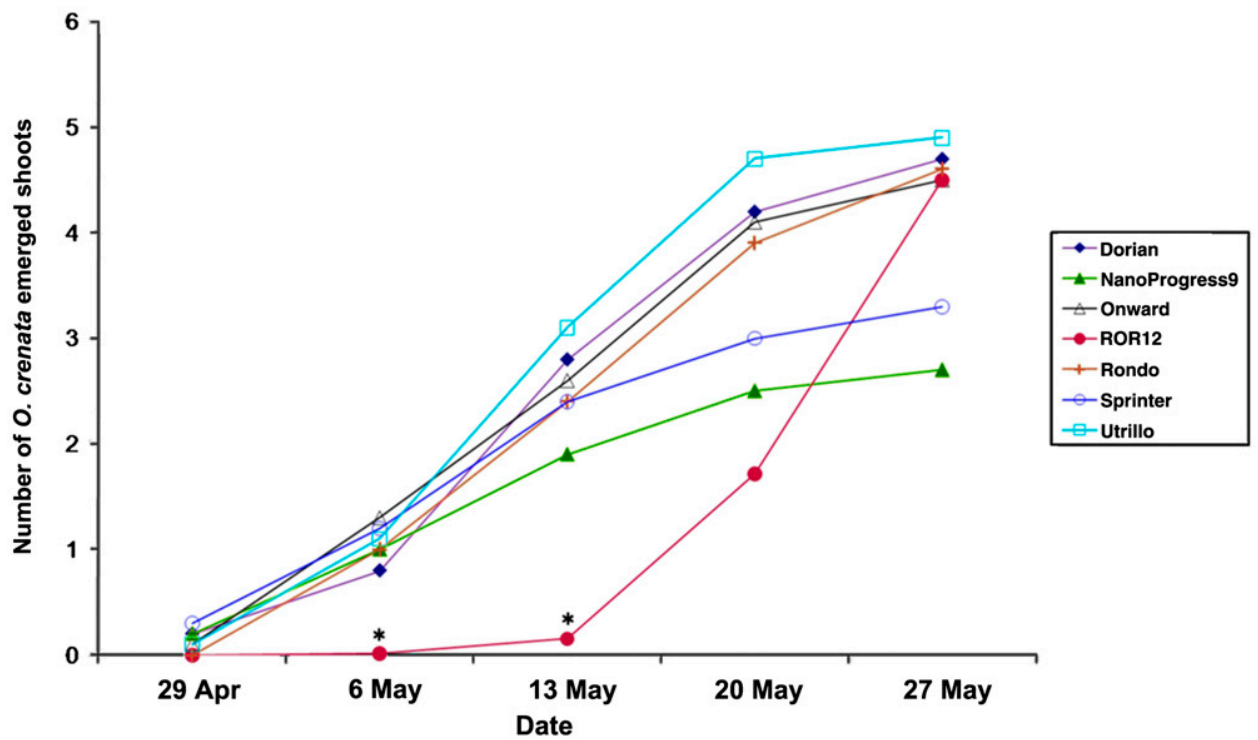

Fig. 2. Temporal trend of Orobanche crenata floral shoot emergence recorded on ROR12 and the susceptible cultivars Dorian, NanoProgress9, Onward, Rondo, Sprinter and Utrillo. Asterisks indicate significant differences between ROR12 and the other genotypes, inferred by the Student's $t$ test $(P<0.05)$. 
provided in this study is not conclusive and, thus, other mechanisms such as differences in germination stimulants other than strigolactones, germination inhibitors, or postgermination defense mechanisms (Perez De Luque et al. 2005) might be also be (co) responsible for the resistant phenotype.

ROR12 induced less (about one-half) $O$. crenata germination than three susceptible cultivars (Fig. 5). This does not correlate one-in-one with the dramatic reduction of the strigolactone content in ROR12 root exudates (Fig. 4). As mentioned above, defense mechanisms not related to strigolactones could also be involved in ROR12 resistance. On the other hand, a nonlinear relationship between pea root exudate strigolactone levels and the $O$. crenata germination rate has been reported before (GomezRoldan et al. 2008). In rice, a difference of at least 50-fold in strigolactone concentration in the root exudates of NERICA rice varieties corresponded with a difference in germination percentage of only, at most, eightfold (Jamil et al. 2011). Similarly, Yoneyama et al. (2010) found that an approximately 40-fold difference in the production of the strigolactone 5-deoxystrigol in rice genotypes was associated with a moderate reduction of the germination rate of the parasitic weed Striga hermontica.

A moderate difference in the germination-inducing activity of root exudates between susceptible cultivars and ROR12 is also seemingly in contrast with the almost complete lack of O. crenata emergence on ROR 12 in three out of four field trials carried out in this study (Table 1). This might be explained by insufficient time for the parasite to emerge aboveground before crop maturity, as it was shown that ROR12 resistance is also correlated with delayed emergence of $O$. crenata (Fig. 2). The delayed emergence of $O$. crenata observed on ROR 12 may be the consequence of the lower induction of parasite seed germination due to a lower concentration of strigolactones in the root exudate.

Prior to this work, the rms 1 and $r m s 5$ mutants were the only strigolactone-deficient genotypes in pea to be characterized (Gomez-Roldan et al. 2008). Although both of them induce less germination of $O$. crenata than the corresponding wild type, they exhibit severe pleiotropic phenotypes, in particular, dwarfism and extreme branching that make them unsuitable for breeding purposes (Rameau et al. 1997). Although ROR12 also exhibits a branched phenotype, we showed that its yield potential is comparable to that of modern cultivars (Fig. 3), thus suggesting that it is a suitable genotype to be exploited in breeding programs for broomrape resistance. We speculate that the low level of strigolactones that is still present in ROR12 is sufficient to prevent severe pleiotropic effects that are manifest in case of a complete lack of strigolactones, such as in the rms mutants.

Low strigolactone-producing cultivars displaying resistance to parasitic weeds have been described in rice and, recently, in faba bean (Fernández-Aparicio et al. 2014; Jamil et al. 2011). Together with the results of the present work, this indicates that the selection of genotypes impaired in genes involved in strigolactone biosynthesis or exudation might be a suitable breeding target for cultivated species affected by parasitic weeds.

Previous studies pointed out that phosphorous limitation leads to increased release of strigolactones in the rhizosphere (López-Ráez et al. 2008; Yoneyama et al. 2007). This was confirmed by our study, as LC-MS/MS analysis of root exudates suggested a positive correlation between duration of phosphorous starvation and strigolactone levels. However, such a correlation appeared to be genotype-dependent, as it was found to be much stronger for the commercial cultivar Sprinter than in the other genotypes investigated (Fig. 4). This indicates that the selection of genotypes for which strigolactone biosynthesis is less influenced by phosphorous starvation might also represent a breeding strategy for parasitic weed resistance.

At the moment, we are developing a population of segregating recombinant inbred lines, with the goal of molecular characterization of the novel resistance source reported here. We anticipate that this will enhance our understanding of the basis of parasitic plant resistance and, possibly, the biology of strigolactones. Moreover, it might lead to the identification of genes suitable to be targeted by reverse genetics approaches, with the objective to identify either parasitic weed resistance, low-strigolactone production, or both in crop species affected by root parasitic plants.

\section{1-2012}

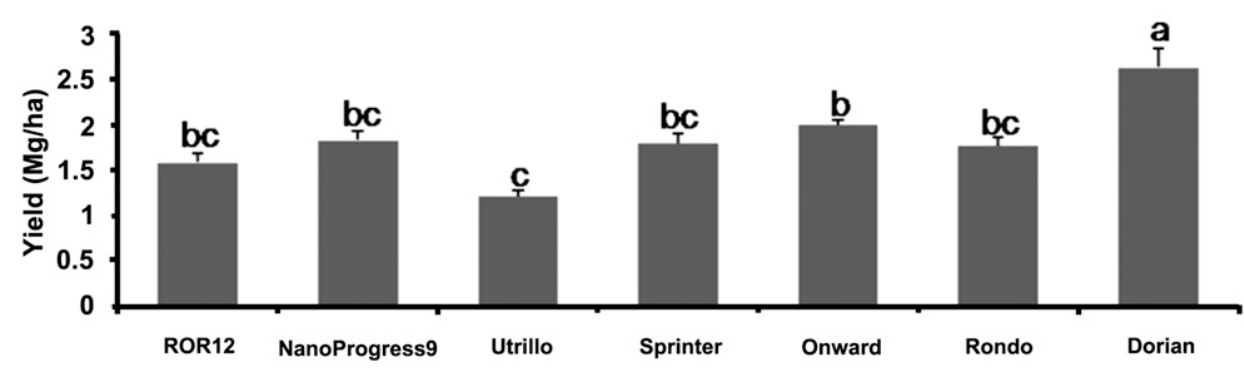

2012-2013

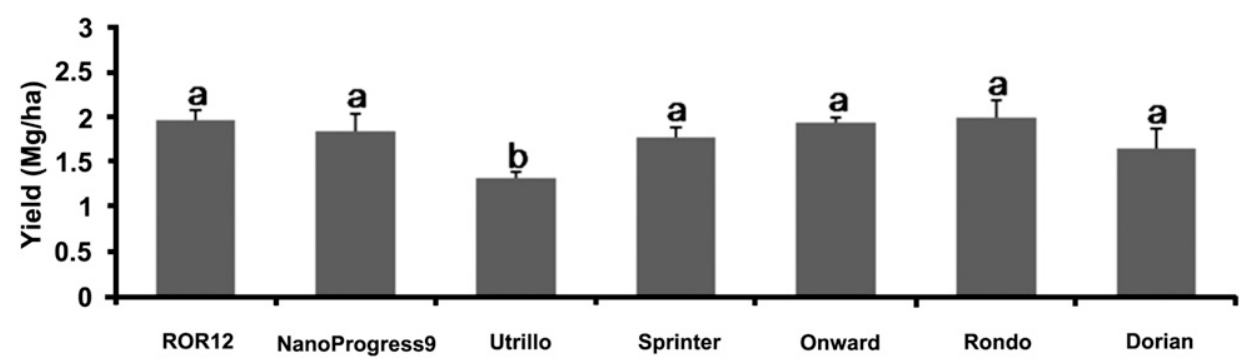

Fig. 3. Yield of the ROR12 line and cultivars Dorian, NanoProgress9, Onward, Rondo, Sprinter, and Utrillo recorded during the growing seasons 2011-2012 and 2012-2013 in experimental fields not infested by $O$. crenata. Different letters indicate significant differences between the treatments, inferred by the Student Newmann Keuls test $(P<0.05)$. The error bars denote standard errors. 


\section{MATERIALS AND METHODS}

Selection of the resistant line ROR12.

A garden pea landrace was selected during a germplasm screening for being resistant to $O$. crenata. Single plants sampled from this landrace were further subjected to repeated self-pollination and corresponding lines were evaluated in experimental fields known to be severely infested with $O$. crenata seeds. A resistant line, named ROR12, was finally extracted.

\section{Plant material.}

Garden pea 'Sprinter', 'NanoProgress9', 'Utrillo', 'Onward', 'Rondo', and 'Dorian', available in the pea germplasm collection of the Department of Soil, Plant and Food Science of the
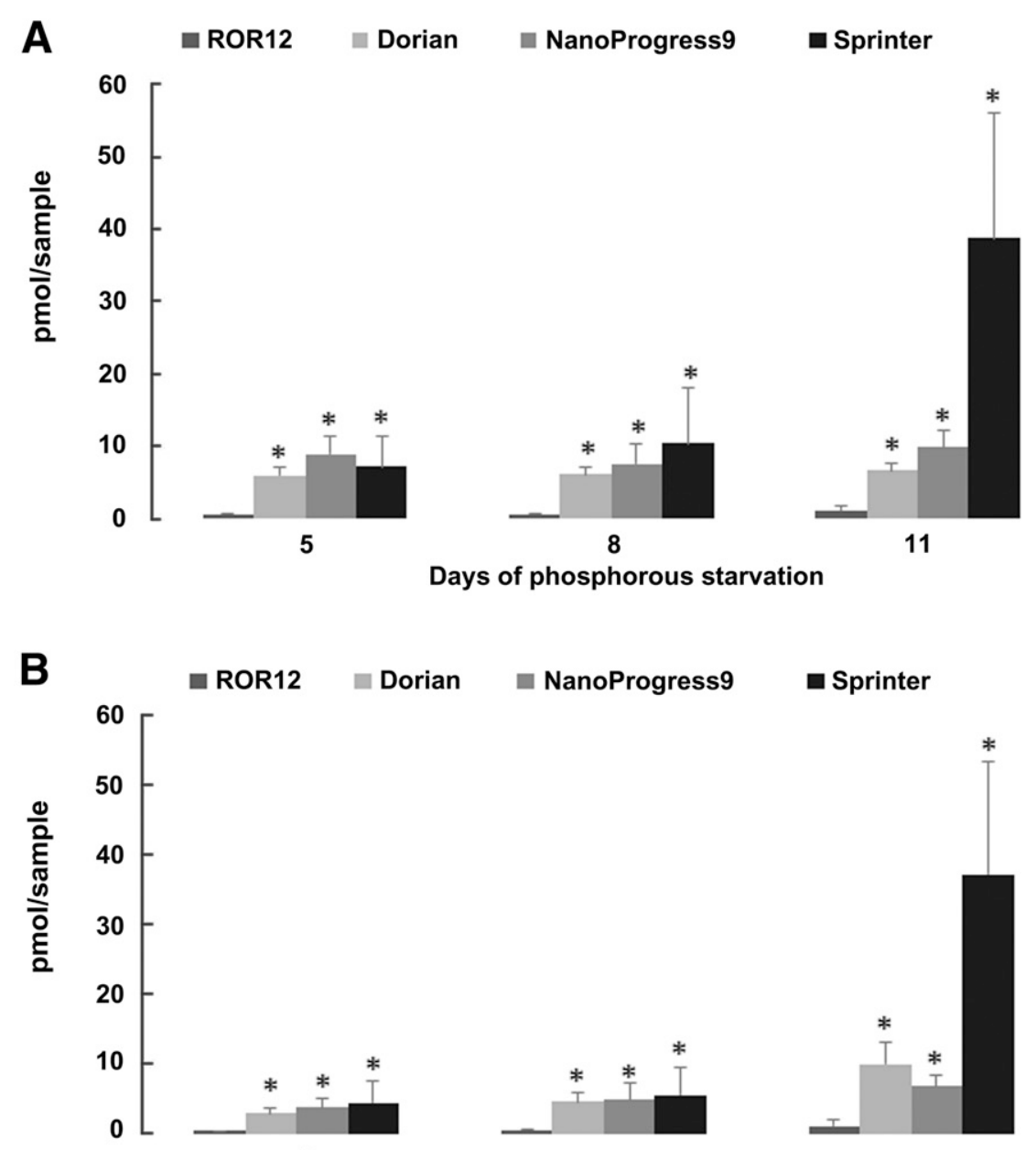

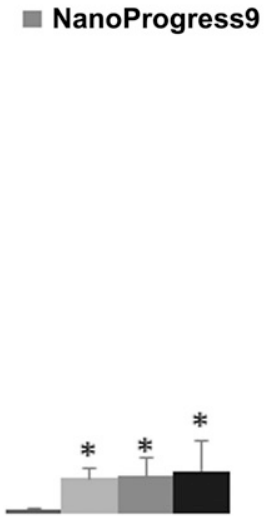

8

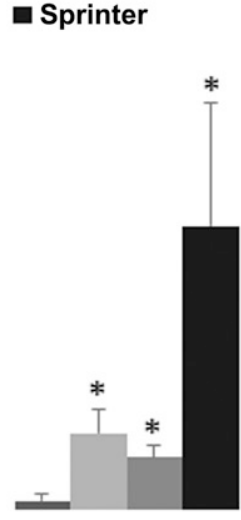

11

Days of phosphorous starvation

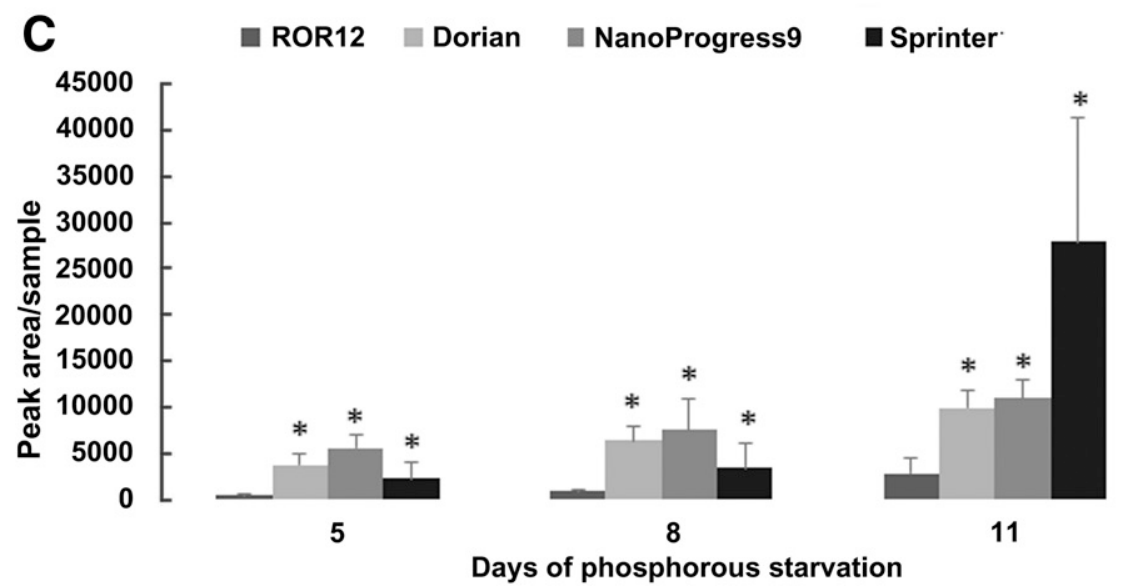

Fig. 4. Levels of the three strigolactones, A, orobanchol, B, orobanchyl acetate, and C, fabacyl acetate, detected in pea root exudates of the ROR12 line selected in this study compared with three cultivars susceptible to Orobanche crenata. Individual samples refer to 5 liters of root exudates collected from 14 plants in $24 \mathrm{~h}$. Orobanchol and orobanchyl acetate levels are expressed in picomoles per sample. Relative differences in the levels of fabacyl acetate, for which an authentic standard was not available, are expressed in terms of peak area. Asterisks indicate significant differences between ROR 12 and the other genotypes, inferred by performing Student's $t$ test $(P<0.05)$. The error bars denote standard errors. 
University of Bari (Italy), were used in this study, together with the selected resistant line ROR12. O. crenata seeds used for germination bioassays were collected from heavily infested pea fields in the area of Bari.

\section{Field trials.}

Four field trials, carried out in three different growing seasons (2009-2010, 2010-2011, and 2012-2013) were set up at the experimental farm "P. Martucci" of the University of Bari, in order to evaluate genotypic responses to O. crenata. All the trials were performed according to a randomized block design, in plots known from previous cultivation of legumes to be severely infested with $O$. crenata seeds. Planting density was set to 1.25 plants per square meter. For each plant, yield and number of $O$. crenata shoots emerging aboveground at five different time points were recorded. In 2009-2010 and 20102011 , single trials were performed, in which the two genotypes ROR12 and 'Sprinter' were compared. In 2012-2013, two trials were carried out, in which the panel of controls was extended to include 'NanoProgress9', 'Utrillo', 'Onward', 'Rondo', and 'Dorian'. The statistical significance of differences between means was assessed using the Student's $t$ test.

To evaluate the yield potential of the ROR12 line, two additional randomized block design trials, in which its performance was compared with the same commercial cultivars mentioned above in plots free of $O$. crenata seeds, were carried out in the growing seasons 2011-2012 and 2012-2013. In this case, plants were sown at common agronomic garden pea field density (45 plants per square meter). Genotypic differences were assessed using the Student Newmann Keuls test.

\section{Root exudate collection.}

Pea seeds from the genotypes 'Sprinter', 'Dorian', 'Progress9', and ROR 12 were germinated for $48 \mathrm{~h}$ on moistened filter paper at $25^{\circ} \mathrm{C}$ in darkness and were then transferred, in a greenhouse, in pots filled with vermiculite for 12 days. Fourteen seedlings of each genotype were transplanted into a X-stream 20 aeroponics system (Nutriculture) operating with 5 liters of modified half-strength Hoagland solution (López-Ráez et al. 2008 ) and were grown under controlled conditions (16 h light, $23^{\circ} \mathrm{C}, 60 \%$ relative humidity), replacing the nutrient solution twice a week. Three biological replicates were used. Two weeks after transplanting, phosphorous starvation was induced by replacing the above-mentioned solution with one without $\mathrm{KH}_{2} \mathrm{PO}_{4}$. Root exudates (5 liters) were collected after 5, 8, and 11 days of phosphorous starvation, refreshing the nutrient solution $24 \mathrm{~h}$ before collection, in order to remove all accumulated strigolactones. In order to purify and concentrate root exudates, these were loaded onto pre-equilibrated C18 SPE Grace pure C18-fast 5,000 mg/20 ml columns (W.R. Grace and Co.). The columns were then washed with $50 \mathrm{ml}$ of demineralized water and $50 \mathrm{ml}$ of $30 \%$ acetone/water. Elution of strigolactones from the columns was carried out with $50 \mathrm{ml}$ of $60 \%$ acetone/water. Root exudate samples $(2 \mathrm{ml})$ were transferred into 4-ml glass vials and, after the addition of $( \pm)^{13} \mathrm{C}_{2}$-strigol as an internal standard at the rate of $100 \mathrm{pmol} / \mathrm{ml}$ in the final sample, were dried in a SpeedVacuum SC100 (Savant Instruments) and, subsequently, were dissolved in $50 \mu \mathrm{l}$ of ethyl acetate, were diluted with $4 \mathrm{ml}$ of hexane, and were stored at $-20^{\circ} \mathrm{C}$ until use.

\section{Strigolactone characterization of root exudates.}

In order to be further purified for LC-MS/MS analysis, root exudate samples were loaded onto preconditioned $200 \mathrm{mg} / 3 \mathrm{ml}$ silica columns (Merk, Germany). Five fractions of $2 \mathrm{ml}$ each were eluted, using the following eluting solutions: $100 \%$ hexane, $80 \%$ hexane $/ 20 \%$ ethyl acetate, $60 \%$ hexane $/ 40 \%$ ethyl acetate, $40 \%$ hexane $/ 60 \%$ ethyl acetate, $20 \%$ hexane $/ 80 \%$ ethyl acetate. After the elution, from each fraction, the solvent was evaporated and the residue was redissolved in $200 \mu \mathrm{l}$ of 25:75: 0.1 acetonitrile/water/formic acid, was filtered with Minisart syringe filters (Sartorius), and was used for LC-MS/MS analysis on a Waters Xevo tandem mass spectrometer (Waters) equipped with an electrospray ionization (ESI) source and coupled to an Acquity UPLC system (Waters). An Acquity UPLC BEH C18 column $(100 \times 2.1 \mathrm{~mm}, 1.7 \mu \mathrm{m})$ was used for chromatographic separation. The following acetonitrile/water gradient was applied to the column: $5 \%$ acetonitrile for $1 \mathrm{~min}$, rising to $25 \%$ acetonitrile for $2 \mathrm{~min}$, followed by a 7.5 -min gradient to $35 \%$ acetonitrile and, then, a 2.5 -min gradient to $65 \%$ acetonitrile and 1-min gradient to $90 \%$ acetonitrile, which was maintained for $1.7 \mathrm{~min}$, and finally, back to $5 \%$ acetonitrile, using a 0.3-min gradient. The column, previously equilibrated with the solvent solution for $3 \mathrm{~min}$, was operated

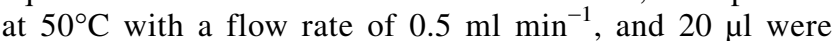
injected for each sample.

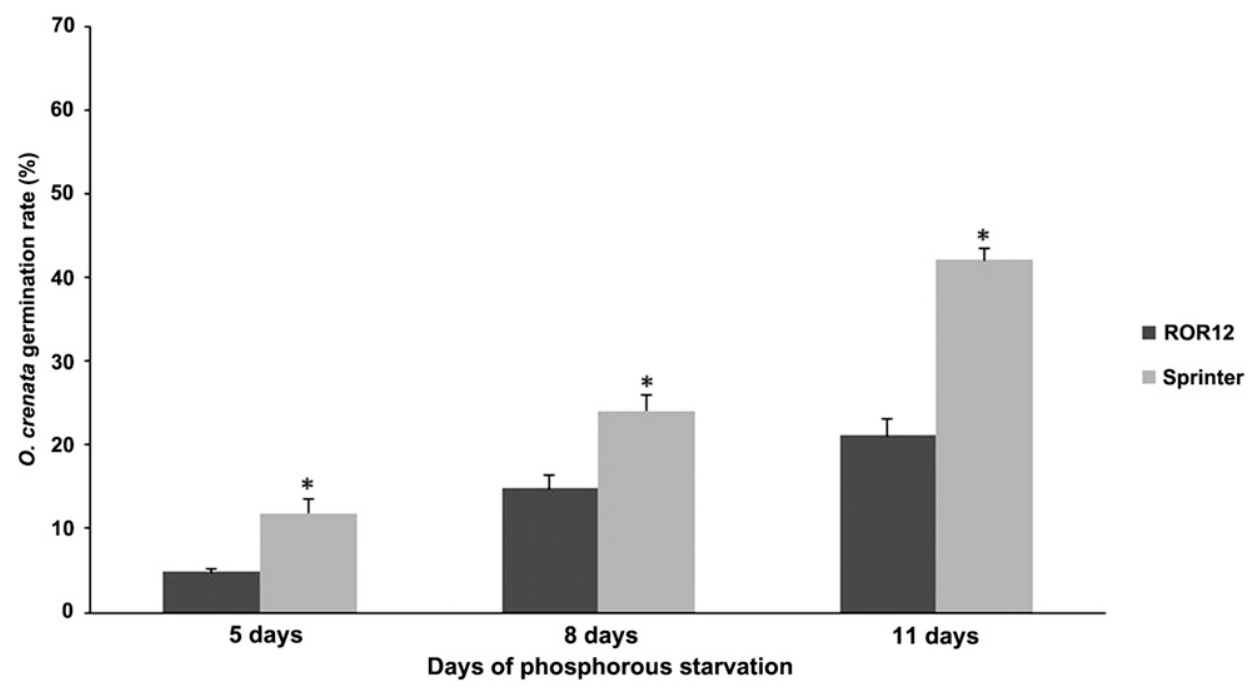

Fig. 5. Germination of Orobanche crenata seeds induced by root exudates of the resistant line ROR 12 and the susceptible cultivar Sprinter, collected at different time points after initiation of phosphorous starvation $(5,8$, and 11 days). Asterisks indicate significant differences, inferred by the Student's $t$ tests $(P<$ 0.05). The error bars denote standard errors. 
The mass spectrometer was operated in positive ESI mode with cone and desolvation gas flows set to 50 and 1,000 liters $\mathrm{h}^{-1}$, respectively. Capillary was set at $3.0 \mathrm{kV}$, the source temperature at $150^{\circ} \mathrm{C}$, and desolvation temperature at $650^{\circ} \mathrm{C}$. The identification of strigolactone molecules was achieved by multiple reaction monitoring (MRM) by mean of a comparison of the retention time and the MRM mass transition with those of the authentic strigolactones orobanchol, 2'-epi-orobanchol and orobanchyl acetate. Fabacyl acetate, for which no authentic standard was available, was identified by using a pea exudate fraction known to contain this compound, which was prepared before (Gomez-Roldan et al. 2008). For orobanchyl acetate and fabacyl acetate, which were found in both $60 \%$ hexane $/ 40 \%$ ethyl acetate and $40 \%$ hexane $/ 60 \%$ ethyl acetate elutions, the quantification was carried out considering the sum of the two fractions.

The MRM channels used for detection and quantification of orobanchol were $\mathrm{m} / \mathrm{z} .347 .2>205.2$ and $\mathrm{m} / \mathrm{z} .347 .2>97$; for detection and quantification of orobanchyl acetate $\mathrm{m} / z, 389.15>$ 347.2 and $\mathrm{m} / \mathrm{z}, 389.15>233.15$; for detection and quantification of fabacyl acetate $\mathrm{m} / \mathrm{z} 405.2>231$ and $\mathrm{m} / \mathrm{z} 405.2>97$; for detection and quantification of ${ }^{13} \mathrm{C}_{2}$-strigol used as internal standard $m / z, 349.2>217.2$ and $m / z 349.2>97$. Other channels were used for detection of other SLs and are not shown here because no other known strigolactones were detected in pea. MassLynx 4.1 (Waters) was used for data acquisition and analysis.

\section{Determination of root dry weight.}

Ten plants of the genotypes ROR12, 'Sprinter', and 'Progress9' were grown into a X-stream 20 aeroponics system (Nutriculture) under controlled conditions $\left(16 \mathrm{~h}\right.$ of light, $21^{\circ} \mathrm{C}$, $60 \%$ relative humidity). After four weeks, roots from each plant were collected and were dried at $105^{\circ} \mathrm{C}$ for $24 \mathrm{~h}$. Dry root weight differences between ROR12 and the other two genotypes were assessed using the Student's $t$ test.

\section{O. crenata germination bioassay.}

Aliquots of 'Sprinter' and ROR12 root exudates used for LC-MS/MS analysis $(50 \mu \mathrm{l})$ were concentrated five-, 10-, and 25fold and were applied to $1-\mathrm{cm}$ glass filter paper disks containing approximately $30 \mathrm{O}$. crenata seeds. These had been previously sterilized for $5 \mathrm{~min}$ in $50 \mathrm{ml}$ of $2 \%$ bleach solution with $100 \mu \mathrm{l}$ of Tween20, washed three times with sterile demineralized water, dried superficially, and finally, had been preconditioned at $23^{\circ} \mathrm{C}$ in darkness for 2 weeks. Each treatment (genotype $\times$ time point of phosphorous starvation $\times$ root exudate concentration) was replicated three times. A treatment with demineralized water was included as negative control. After 7 days at $21^{\circ} \mathrm{C}$ in darkness, the ratio of germinated to nongerminated seeds was assessed, using a stereomicroscope. Germination rates were subjected to arcsine (square $\operatorname{root}[x]$ ) transformation and significant differences were assessed using the least significant difference test.

\section{ACKNOWLEDGMENTS}

We are grateful to K. Yoneyama for providing strigolactone standards.

\section{LITERATURE CITED}

Akiyama, K., Matsuzaki, K., and Hayashi, H. 2005. Plant sesquiterpenes induce hyphal branching in arbuscular mycorrhizal fungi. Nature 435:824-827.

Brewer, P. B., Koltai, H., and Beveridge, C. A. 2013. Diverse roles of strigolactones in plant development. Mol. Plant 6:18-28.

Cardoso, C., Ruyter-Spira, C., and Bouwmeester, H. J. 2011. Strigolactones and root infestation by plant-parasitic Striga, Orobanche and Phelipanche spp. Plant Sci. 180:414-420.

Cook, C. E., Whichard, L. P., Turner, B., Wall, M. E., and Egley, G. H. 1966. Germination of witchweed (Striga lutea Lour.): Isolation and properties of a potent stimulant. Science 154:1189-1190.
Fernández-Aparicio, M., Kisugi, T., Xie, X., Rubiales, D., and Yoneyama, K. 2014. Low strigolactone root exudation: A novel mechanism of broomrape (Orobanche and Phelipanche spp.) resistance available for faba bean breeding. J. Agric. Food Chem. 62:7063-7071.

Gomez-Roldan, V., Fermas, S., Brewer, P. B., Puech-Pagès, V., Dun, E. A., Pillot, J. P., Letisse, F., Matusova, R., Danoun, S., Portais, J. C., Bouwmeester, H., Bécard, G., Beveridge, C. A., Rameau, C., and Rochange, S. F. 2008. Strigolactone inhibition of shoot branching. Nature 455:189-194.

Jamil, M., Rodenburg, J., Charnikhova, T., and Bouwmeester, H. J. 2011. Pre-attachment Striga hermonthica resistance of New Rice for Africa (NERICA) cultivars based on low strigolactone production. New Phytol 192:964-975

Joel, D. M., Gressel, J., and Musselman, L. J., eds. 2013. Parasitic Orobanchaceae: Parasitic mechanisms and control strategies. SpringerVerlag, Berlin.

Kohlen, W., Charnikhova, T., Lammers, M., Pollina, T., Tóth, P., Haider, I., Pozo, M. J., de Maagd, R. A., Ruyter-Spira, C., Bouwmeester, H. J., and López-Ráez, J. A. 2012. The tomato CAROTENOID CLEAVAGE DIOXYGENASE8 (SlCCD8) regulates rhizosphere signaling, plant architecture and affects reproductive development through strigolactone biosynthesis. New Phytol. 196:535-547.

López-Ráez, J. A., Charnikhova, T., Gómez-Roldán, V., Matusova, R., Kohlen, W., De Vos, R., Verstappen, F., Puech-Pages, V., Bécard, G., Mulder, P., and Bouwmeester, H. 2008. Tomato strigolactones are derived from carotenoids and their biosynthesis is promoted by phosphate starvation. New Phytol. 178:863-874.

Pavan, S., Schiavulli, A., Appiano, M., Miacola, C., Visser, R. G. F., Bai, Y., Lotti, C., and Ricciardi, L. 2013. Identification of a complete set of functional markers for the selection of $\mathrm{er} 1$ powdery mildew resistance in Pisum sativum L. Mol. Breed. 31:247-253.

Perez-De-Luque, A., Jorrin, J., Cubero, J. I., and Rubiales, D. 2005. Orobanche crenata resistance and avoidance in pea (Pisum spp.) operate at different developmental stages of the parasite. Weed Res. 45:379-387.

Rameau, C., Bodelin, C., Cadier, D., Grandjean, O., Miard, F., and Murfet, I. C. 1997. New ramosus mutants at loci Rms1, Rms3 and Rms4 resulting from the mutation breeding program at Versailles. Pisum Genet. 29:7-12.

Rubiales, D., Fernández-Aparicio, M., Pérez-de-Luque, A., Castillejo, M. A., Prats, E., Sillero, J. C., Rispail, N., and Fondevilla, S. 2009 Breeding approaches for crenate broomrape (Orobanche crenata Forsk.) management in pea (Pisum sativum L.). Pest Manag. Sci. 65: 553-559.

Rubiales, D., Moreno, M. T., and Sillero, J. C. 2005. Search for resistance to crenate broomrape (Orobanche crenata Forsk.) in pea germplasm. Genet. Resour. Crop Evol. 52:853-861.

Rubiales, D., Perez-de-Luque, A., Cubero, J. I., and Sillero, J. C. 2003. Crenate broomrape (Orobanche crenata) infection in field pea cultivars. Crop Prot. 22:865-872.

Rubiales, D., Perez-de-Luque, A., Fernandez-Aparicio, M., Sillero, J. C., Roman, B., Kharrat, M., Khalil, S., Joel, D. M., and Riches, C. 2006 Screening techniques and sources of resistance against parasitic weeds in grain legumes. Euphytica 147:187-199.

Ruyter-Spira, C., Al-Babili, S., van der Krol, S., and Bouwmeester, H. 2013. The biology of strigolactones. Trends Plant Sci. 18:72-83.

Umehara, M., Hanada, A., Yoshida, S., Akiyama, K., Arite, T., TakedaKamiya, N., Magome, H., Kamiya, Y., Shirasu, K., Yoneyama, K. Kyozuka, J., and Yamaguchi, S. 2008. Inhibition of shoot branching by new terpenoid plant hormones. Nature 455:195-200.

Van Ast, A., and Bastiaans, L. 2006. The role of infection time in the differential response of sorghum cultivars to Striga hermonthica infection. Weed Res. 46:264-274.

Vogel, J. T., Walter, M. H., Giavalisco, P., Lytovchenko, A., Kohlen, W., Charnikhova, T., Simkin, A. J., Goulet, C., Strack, D., Bouwmeester, H. J., Fernie, A. R., and Klee, H. J. 2010. S1CCD7 controls strigolactone biosynthesis, shoot branching and mycorrhiza-induced apocarotenoid formation in tomato. Plant J. 61:300-311.

Xie, X., Yoneyama, K., Harada, Y., Fusegi, N., Yamada, Y., Ito, S., Yokota, T., Takeuchi, Y., and Yoneyama, K. 2009. Fabacyl acetate, a germination stimulant for root parasitic plants from Pisum sativum. Phytochemistry 70:211-215.

Yoneyama, K., Awad, A. A., Xie, X., Yoneyama, K., and Takeuchi, Y. 2010. Strigolactones as germination stimulants for root parasitic plants. Plant Cell Physiol. 51:1095-1103.

Yoneyama, K., Xie, X., Yoneyama, K., and Takeuchi, Y. 2009. Strigolactones: Structures and biological activities. Pest Manag. Sci. 65:467-470.

Yoneyama, K., Yoneyama, K., Takeuchi, Y., and Sekimoto, H. 2007. Phosphorus deficiency in red clover promotes exudation of orobanchol, the signal for mycorrhizal symbionts and germination stimulant for root parasites. Planta 225:1031-1038. 